\title{
TGF- $\beta 1$ conjugated chitosan collagen hydrogels induce chondrogenic differentiation of human synovium-derived stem cells
}

\author{
Jinku Kim ${ }^{1 \dagger}$, Brian Lin ${ }^{2 \dagger}$, Soyon Kim³ ${ }^{3}$ Bogyu Choi ${ }^{2}$, Denis Evseenko ${ }^{4}$ and Min Lee $2,3^{*}$
}

\begin{abstract}
Background: Unlike bone tissue, articular cartilage regeneration has not been very successful and has many challenges ahead. We have previously developed injectable hydrogels using photopolymerizable chitosan (MeGC) that supported growth of chondrocytes. In this study, we demonstrate a biofunctional hydrogel for specific use in cartilage regeneration by conjugating transforming growth factor- $\beta 1$ (TGF- $\beta 1$ ), a well-documented chondrogenic factor, to MeGC hydrogels impregnating type II collagen (Col II), one of the major cartilaginous extracellular matrix (ECM) components.

Results: TGF- $\beta 1$ was delivered from MeGC hydrogels in a controlled manner with reduced burst release by chemically conjugating the protein to MeGC. The hydrogel system did not compromise viability of encapsulated human synovium-derived mesenchymal stem cells (hSMSCs). Col II impregnation and TGF- $\beta 1$ delivery significantly enhanced cellular aggregation and deposition of cartilaginous ECM by the encapsulated cells, compared with pure MeGC hydrogels.
\end{abstract}

Conclusions: This study demonstrates successful engineering of a biofunctional hydrogel with a specific microenvironment tailored to promote chondrogenesis. This hydrogel system can provide promising efficacious therapeutics in the treatment of cartilage defects.

Keywords: Chitosan hydrogels, Type II collagen, Transforming growth factor, Synovium-derived stem cells, Chondrogenic differentiation

\section{Background}

More than 70 million adults in the United States suffer from articular cartilage injuries caused mainly by arthritis. An estimated economic burden to arthritis-related disability would be $\$ 100$ billion by 2020 as elderly population continues to grow [1]. Although autologous chondrocyte implantation (ACI) is clinically available for articular cartilage injuries, successful regeneration of damaged articular cartilage remains a great challenge due to the limitations (e.g. multiple surgical procedures, in vitro cell expansion) associated with ACI [2-5]. Consequently, tissue

\footnotetext{
* Correspondence: leemin@ucla.edu

${ }^{\dagger}$ Equal contributors

2Division of Advanced Prosthodontics, University of California, Los Angeles, CA 90095, USA

${ }^{3}$ Department of Bioengineering, University of California, Los Angeles, CA 90095, USA

Full list of author information is available at the end of the article
}

engineering approaches have been utilized for articular cartilage regeneration [6,7]. However, unlike bone, cartilage regeneration using tissue engineering strategies has not been successful due to unfavorable selection of appropriate cell sources, scaffolds and/or biomolecules to precisely restore structure and function of the elegantly organized tissue $[8,9]$.

Recently, in an effort to remove harsh conditions and chemicals used in scaffold fabrication, our laboratory have developed the chitosan-based hydrogel system by utilizing mild visible blue light (VBL) and riboflavin (vitamin B2) as a light source and an initiator, respectively, instead of popular choices of UV light and harmful photoinitiators $[10,11]$. As a result, the delivery system bio-degraded to match growth rates of cartilage regeneration and removed harsh chemicals while maintaining 
chondrocyte morphology and phenotype, which will be crucial for cartilage regeneration during chondrogenesis of mesenchymal stem cells (MSCs) [12]. Yet, systematic tissue engineering approaches is still required for successful cartilage repair.

Among numerous biomolecules for cartilage repair, transforming growth factor- $\beta 1$ (TGF- $\beta 1$ ) has been explored to enhance chondrogenic differentiation of cartilage forming cells as well as biomechanical properties of neocartilage $[13,14]$. However, therapeutic efficacy of TGF- $\beta 1$ is affected by delivery kinetics due to its intrinsic protein instability and rapid enzymatic degradation in vivo requiring a high therapeutic dose. The high dose requirement can cause adverse side effects such as fibrotic disorders and unwanted osteophyte formation in the synovium $[15,16]$. Sulfated polysaccharides such as heparin have been shown to form stable complexations with TGF- $\beta 1$, which maintain the biological activity and can sustain the protein release [17-20]. Recent studies have used this affinity binding of heparin for the controlled delivery of TGF- $\beta 1$ with prolonged bioactivity [21-23]. However, such a physical adsorption-based delivery approach requires a high amount of growth factors and is easily affected by a local microenvironments causing burst release of the loaded protein. Chemical conjugation of growth factors into scaffolds showed a more prolonged release and significantly reduced initial burse of the proteins compared to physically absorbed growth factors [24,25].

In addition, type II collagen (Col II) is known to be the most abundant protein in the cartilage tissue and to promote chondrogenic differentiation of MSCs [26,27]. Previous studies demonstrated that TGF- $\beta 1$-mediated chondrogenesis was enhanced significantly in the presence of Col II [28-30]. Chondrocytes bind to Col II through integrins leading to the formation of signaling complex that induces chondrogenesis. TGF- $\beta 1$ interacts with a receptor complex and transduces its signals through phosphorylation of the cytoplasmic signaling molecules (Smads). It has been demonstrated that combination of TGF- $\beta 1$ and Col II treatment resulted in a synergistic increase in Smad 2 and 3 phosphorylation compared with the individual stimulation with TGF- $\beta 1$ or Col II alone, indicating the signaling cross-talk between Col II-activated integrin pathway and the TGF- $\beta 1$ Smad pathway [28-30].

Here, we introduce the sophisticated cartilage tissue engineering system supplemented with TGF- $\beta 1$ bioconjugation and Col II impregnation into the methacrylated chitosan (MeGC) hydrogels to promote chondrogenic differentiation of encapsulated MSCs derived from human synovium (hSMSCs). We hypothesized that the TGF- $\beta 1$ conjugation and Col II impregnation into the chitosan hydrogels will additively enhance chondrogenic differentiation of encapsulated hSMSCs. To test this hypothesis, we encapsulated hSMSCs into the MeGC hydrogels functionalized with Col II and TGF- $\beta 1$ and determined the ability of the hydrogel systems to promote chondrogenesis using a series of in vitro assays at different time courses up to 21 days.

\section{Results}

\section{Hydrogel fabrication and characterization}

Chitosan (MeGC) and Col II-impregnated chitosan hydrogels $(\mathrm{MeGC/Col})$ were prepared via free radical polymerization under VBL in the presence of a RF initiator (Figure 1). TGF- $\beta 1$ was chemically conjugated into MeGC prior to hydrogel formation using a Succinimidyl-4-(Nmaleimidomethyl)cyclohexane-1-carboxylate (SMCC) linker (MeGC/Col/TGF). Cross-sectional SEM images of MeGC/ Col suggested that MeGC hydrogels had fibrous collagen microstructure homogeneously distributed throughout the hydrogels (Figure 2B). The conjugation of TGF- $\beta 1$ onto MeGC did not alter the microstructure of the cross-linked hydrogels.

Release of conjugated TGF- $\beta 1$ from MeGC hydrogels was measured in a culture medium containing serum. As expected, the release profiles from the hydrogels showed a sustained release with a reduced initial burst and approximate cumulative release of $12 \%$ up to 21 days (Figure 2C). The addition of Col II did not significantly affect the release kinetics of TGF- $\beta 1$ from the hydrogel (Figure 2D). Our previous data showed that approximately $60 \%$ of initially loaded TGF- $\beta 1$ by an adsorption method was rapidly released from the hydrogels at day one in a similar condition (Additional file 1: Figure S1). Chemically conjugated TGF- $\beta 1$ was better immobilized on the hydrogels, thus much less amount of the protein was released from the delivery system, compared with weakly adsorbed TGF- $\beta 1$. SMCC is one of the widely used crosslinking agents containing an amine-reactive N-hydroxysuccinimide (NHS) ester and a sulfhydrylreactive maleimide group [31]. NHS esters react with the primary amino groups $(-\mathrm{NH} 2)$ of $\mathrm{MeGC}$ to form stable amide bonds. Maleimide groups form covalent crosslinks with sulfhydryl $(-\mathrm{SH})$ moieties on cysteine residues of TGF- $\beta 1$ to form stable thioether bonds. A similar conjugation technique using SMCC was successfully employed to immobilize various growth factors, including latent TGF- $\beta 1$, bone morphogenetic protein-2 (BMP-2), and vascular endothelial growth factor (VEGF) onto biomaterial surfaces, and the conjugated growth factors maintained their bioactivity [24,32-34]. However, the conjugation process and chemistry of reaction can adversely affect the growth factor by protein denaturation and impair the biological function of the growth factor. Additional studies on protein conformational changes are needed for the future clinical translation. 


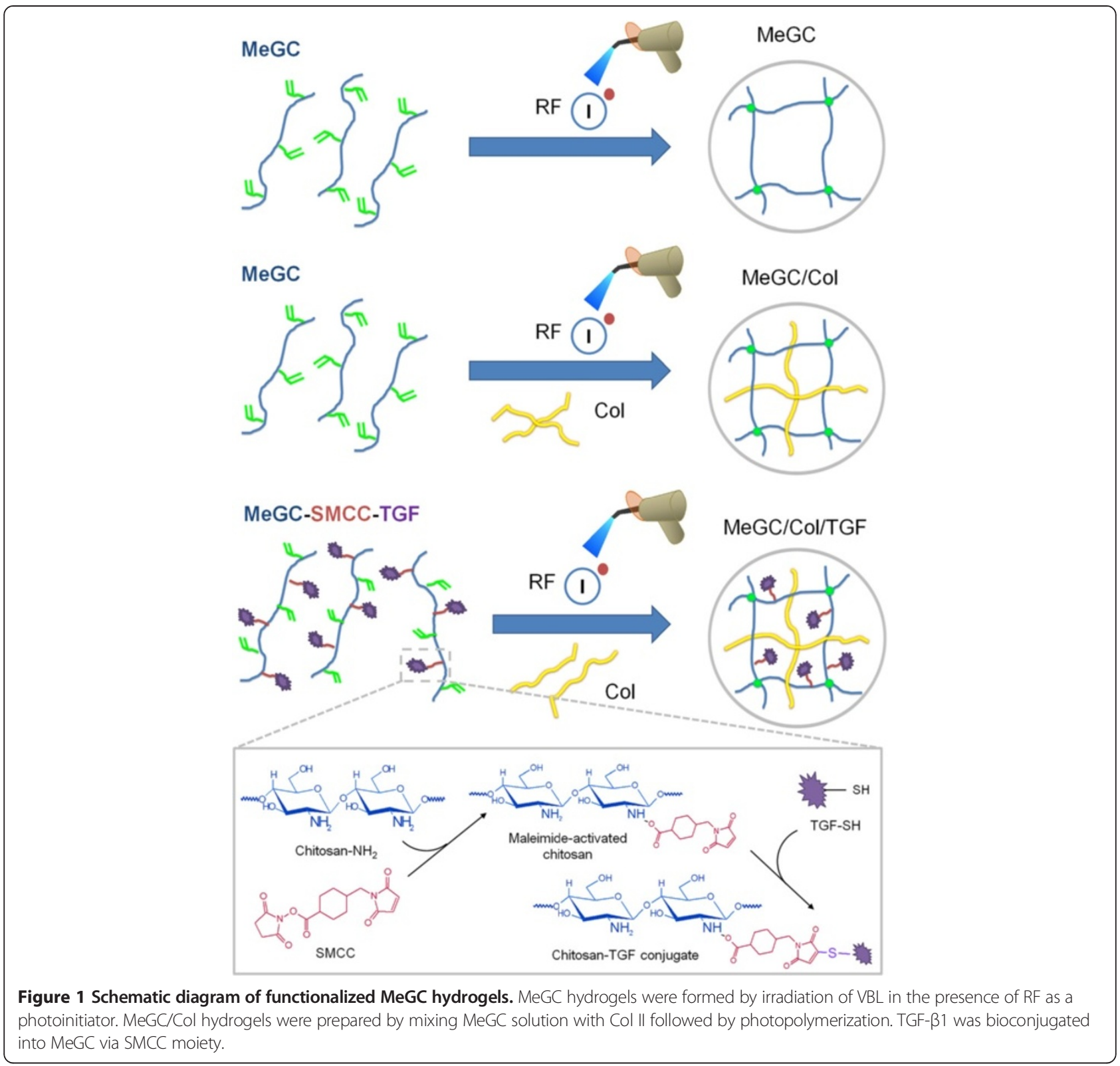

\section{Cell growth in MeGC/Col/TGF hydrogels}

The bright field microscopic images of encapsulated hSMSCs cultured in MeGC, MeGC/Col, or $\mathrm{MeGC/Col} /$ TGF hydrogels showed that there was little cell aggregation at day 1 regardless of the presence of Col II and/or TGF- $\beta 1$ (Figure 3A). Cell aggregation was rarely observed in MeGC hydrogels over the 21-day culture period. In contrast, MeGC/Col hydrogels appeared to have a few clusters at day 7 and the area of cell clusters was increased over time. For TGF- $\beta 1$ conjugated gels, much bigger cell clusters were observed after 7 days compared with other hydrogels. The image analysis data of cell cluster area in each hydrogel confirmed that there were significant differences in cell cluster area between treatment groups and pure MeGC groups at day 7, 14 and 21 days (Figure 3B). In particular, there were significant differences between $\mathrm{MeGC} / \mathrm{Col}$ and $\mathrm{MeGC} / \mathrm{Col} / \mathrm{TGF}$ hydrogels at day 21 , indicating that the conjugated TGF- $\beta 1$ significantly promoted cell aggregations in the hydrogels. Regarding cellularity of encapsulated hSMSCs, the data revealed that $\mathrm{MeGC/Col}$ gels had greatest cellularity as compared to other hydrogels at days 14 and $21 . \mathrm{MeGC} / \mathrm{Col} /$ TGF hydrogels had significant increase of cell proliferation, compared with the MeGC hydrogels (Figure 3C).

The Live/Dead staining images showed that most cells encapsulated in the all tested hydrogels (>90\%) were 

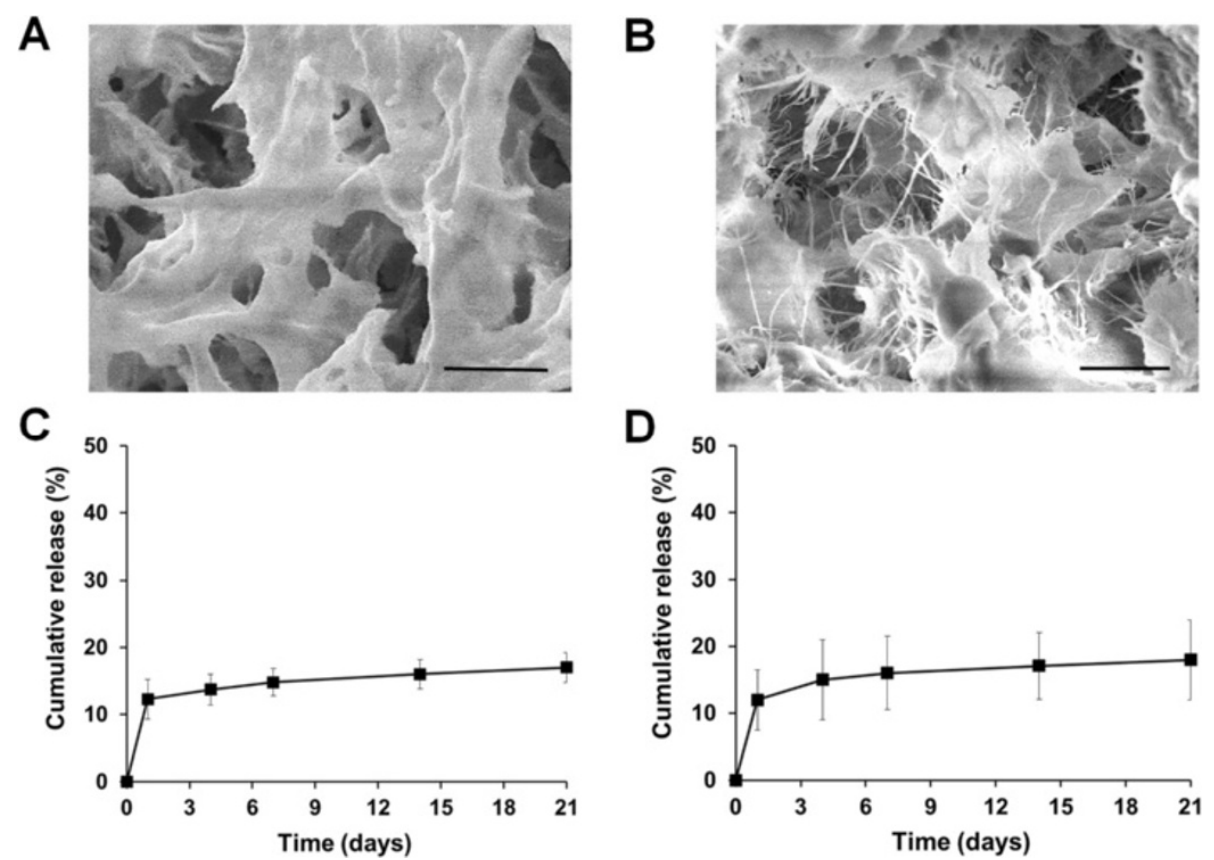

Figure 2 Hydrogel characteristics and release kinetics. SEM images of MeGC (A) and MeGC/Col (B) hydrogels. Scale bar $=10 \mu \mathrm{m}$. Release profiles of TGF- $\beta 1$ from MeGC/TGF (C) and MeGC/Col/TGF (D) hydrogels incubated in culture media with serum.

viable even at day 21 and there were no significant differences in cell viability among tested hydrogel systems, indicating that the use of SMCC, VBL, and $\mathrm{RF}$ all together had no adverse effects on cell viability (Figure 4).

\section{Chondrogenic differentiation of encapsulated hSMSCs}

To observe in vitro chondrogenic differentiation of encapsulated hSMSCs, histology (H\&E staining and Safranin-O staining) and immunohistochemistry (Col II staining) were performed in this study.
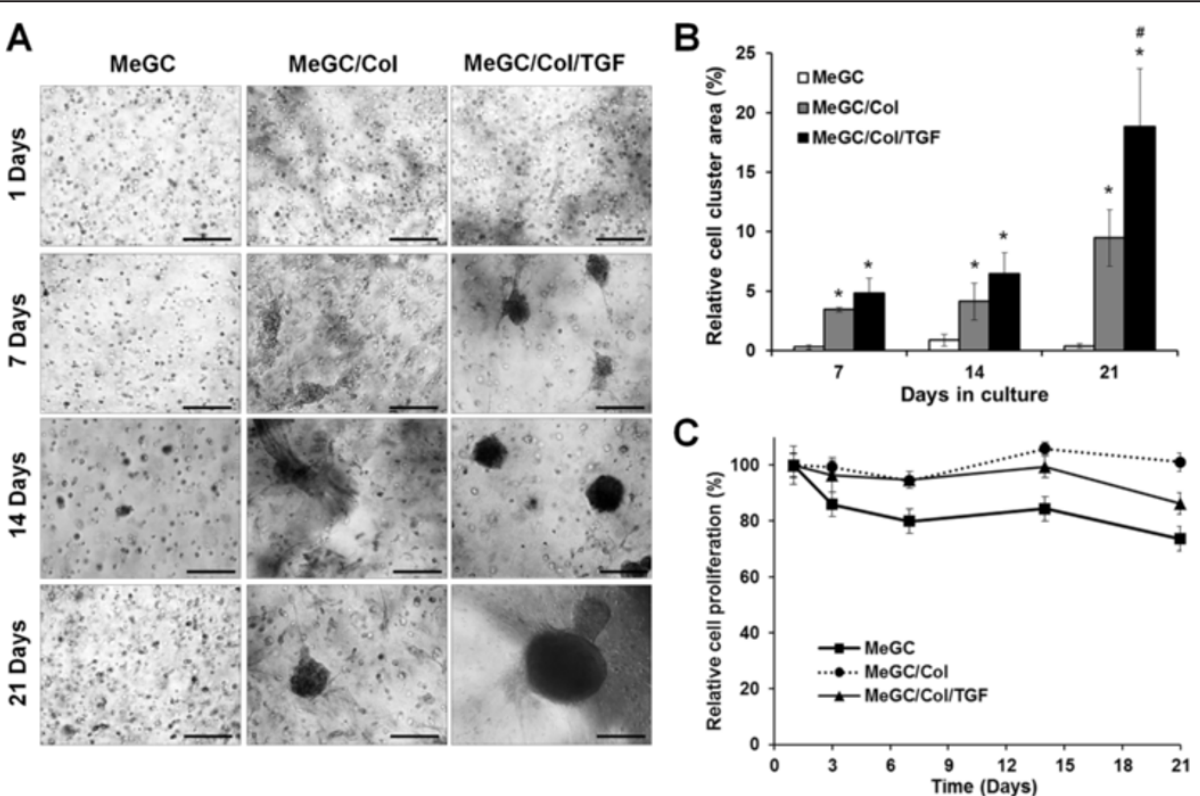

Figure 3 Growth of hSMSCs cultured in hydrogels. (A) Bright field images of hSMSCs in the hydrogels. Scale bar $=200 \mu \mathrm{m}$. (B) Percentage area of cell clusters quantified by image analysis of bright field images. (*: $p<0.05$ compared with MeGC and \#: $p<0.05$ compared with MeGC/Col, $\mathrm{n}=3)(\mathbf{C})$ Proliferation of hSMSCs in hydrogels measured by CCK assay. 

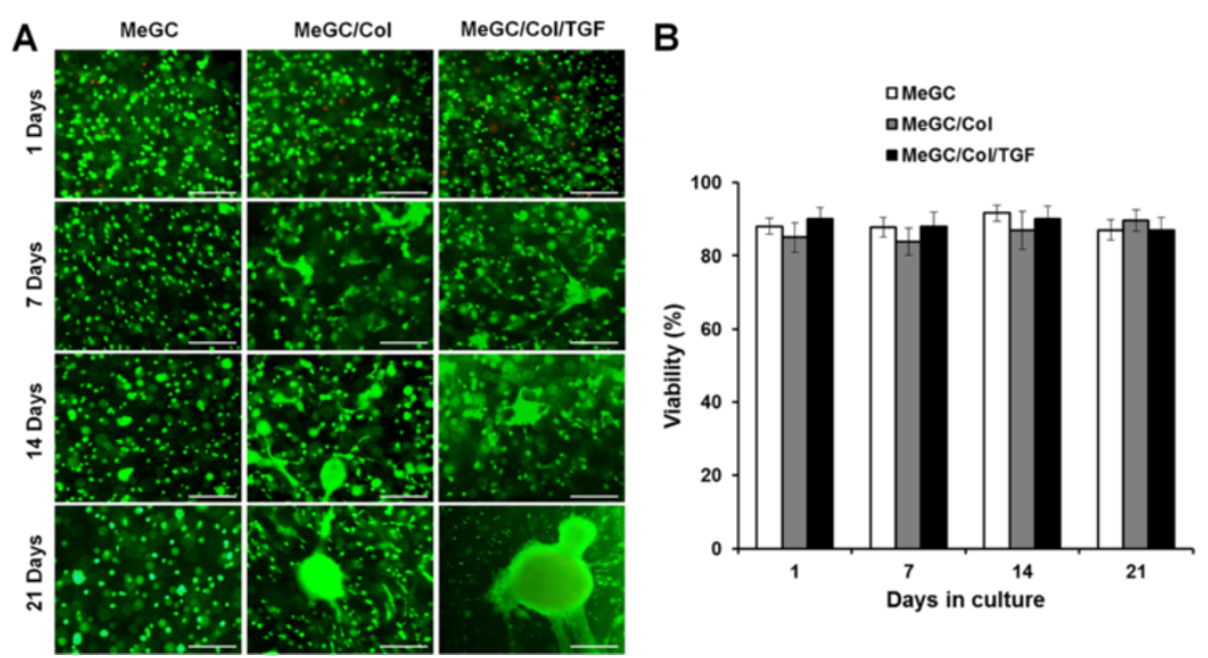

Figure 4 Viability of hSMSCs cultured in hydrogels. (A) Live/Dead staining of hSMSCs in the hydrogels and (B) hSMSCS viability (\%) from live/dead image analysis. Scale bar $=200 \mu \mathrm{m}$.

H\&E staining images revealed homogeneous cell distribution and most of encapsulated cells were round shape with surrounding lacunae in all tested hydrogels at day 7 , which is the characteristic of chondrogenic differentiation (Figure 5). Over the culture period up to day 21 , there were no notable differences in cellular activities in MeGC hydrogels. However, cellular aggregations were observed in $\mathrm{MeGC} / \mathrm{Col}$ and $\mathrm{MeGC/Col} / \mathrm{TGF}$ gels with more intense cell clusters in TGF- $\beta 1$-conjugated gels than $\mathrm{MeGC/Col} \mathrm{gels.}$

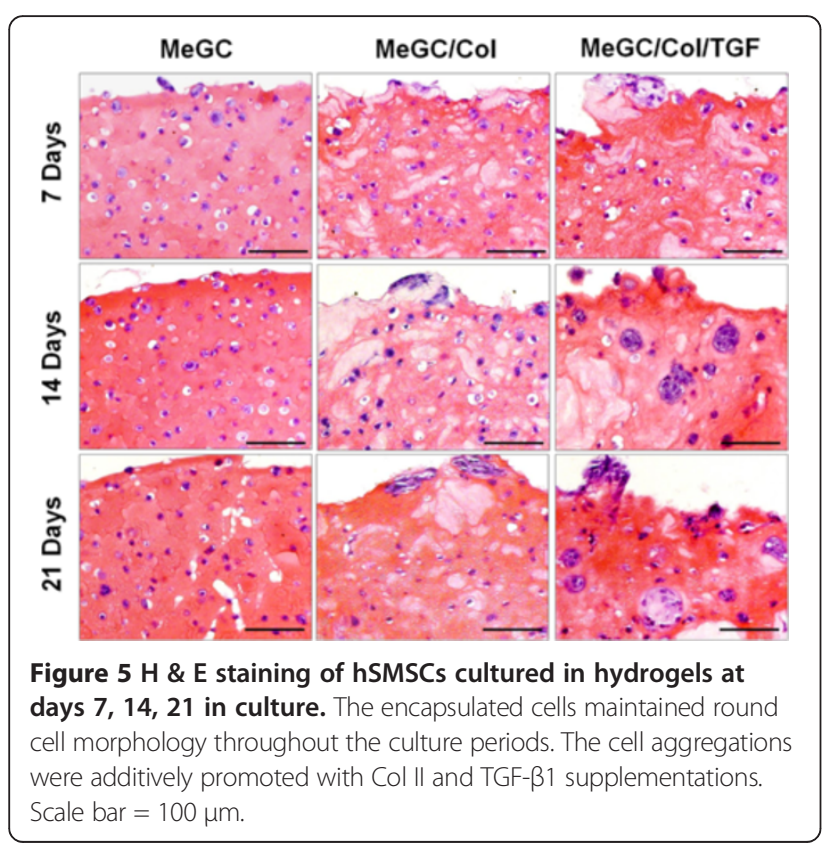

To assess chondrogenic differentiation of encapsulated hSMSCs, safranin-O staining and Col II immunohistochemistry (IHC) were performed to detect sulfated glycosaminoglycan (sGAG) production and Col II deposition from the encapsulated cells. Increased GAG accumulation was observed over time in MeGC/Col and MeGC/ Col/TGF gels, whereas pure MeGC gels did not show any differences in Safranin-O staining density over time (Figure 6A). Quantification of GAG accumulation by image analysis confirmed that there were significant differences between MeGC gels and MeGC/Col/TGF gels at all time periods and between $\mathrm{MeGC} / \mathrm{Col}$ gels and $\mathrm{MeGC} / \mathrm{Col} / \mathrm{TGF}$ gels at day 21 (Figure 6B).

Regarding Col II IHC at day 21, all tested groups showed positive staining for Col II (Figure 7A). In MeGC gels, Col II positive staining was observed throughout the hydrogels but limited inside lacunae, whereas the Col II staining region was extended into the area surrounding the lacunae in $\mathrm{MeGC} / \mathrm{Col}$ gels. Highly intense positive Col II staining was found in $\mathrm{MeGC/Col} / \mathrm{TGF}$ gels compared with $\mathrm{MeGC/Col}$ gels. Quantification of Col II staining verified these findings that Col II expression of encapsulated hSMSCs was significantly higher in $\mathrm{MeGC/Col}$ and MeGC/Col/TGF gels at day 21, compared with MeGC gels (Figure 7B).

The cellular activities of encapsulated hSMSCs in MeGC gels revealed that delivery of TGF- $\beta 1$ without Col II impregnation in MeGC gels did not show any significant effects on cellular activities, compared with pure MeGC gels without TGF- $\beta 1$ (Additional file 2: Figure S2).

Chondrogenic differentiation of hSMSCs cultured in the hydrogels was further confirmed by qRT-PCR analysis (Figure 8). hSMSCs cultured in pellets were used as a 

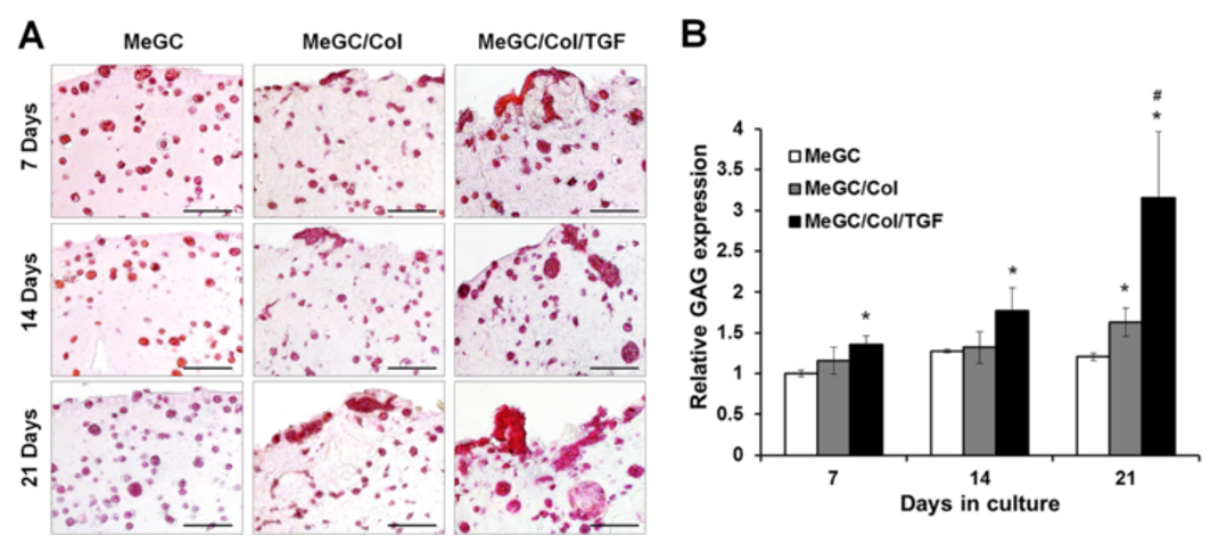

Figure 6 Differentiation of hSMSCs cultured in hydrogels. (A) Safranin-O staining of hSMSCs cultured in hydrogels and (B) quantification of Safranin-O staining by image analysis. Scale bar $=100 \mu \mathrm{m}$. (*: $p<0.05$ compared with MeGC and \#: $p<0.05$ compared with MEGC/Col, $\mathrm{n}=3$ ).

positive control, as the pellet culture system is widely used to induce chondrogenesis of MSCs [35-37]. MeGC/Col/ TGF gels significantly increased the expression level of chondrogenic gene markers Sox 9, aggrecan, and Col II in hSMSCs, relative to MeGC gels. mRNA level of Col II was increased 2.5-fold in the $\mathrm{MeGC/Col} / \mathrm{TGF}$ gels compared to the MeGC gels, whereas no significant change was observed in the MeGC/Col or MeGC/TGF gels.

\section{Discussion}

The primary purpose of this study is to assess whether Col II nanofiber impregnation and TGF- $\beta 1$ bioconjugation into MeGC hydrogels contribute chondrogenic differentiation of encapsulated hSMSCs in the gels. The results of the current study support the hypothesis that the Col II impregnation and TGF- $\beta 1$ conjugation into the chitosan hydrogels additively promoted chondrogenic differentiation of the encapsulated hSMSCs.

Although our previous report demonstrated that MeGC-based hydrogels prepared by VBL and RF, proven to be biocompatible and to promote ECM production (e.g., GAG accumulation) of encapsulated MSCs, [10] those matrix synthesis may be minimal, thus the supplementation of exogenous growth factors or recruiting endogenous ones may be needed to facilitate chondrogenesis $[38,39]$. Among soluble biomolecules to enhance cartilage formation, TGF- $\beta 1$ was selected in this study due to its well-known chondrogenic potentials
A

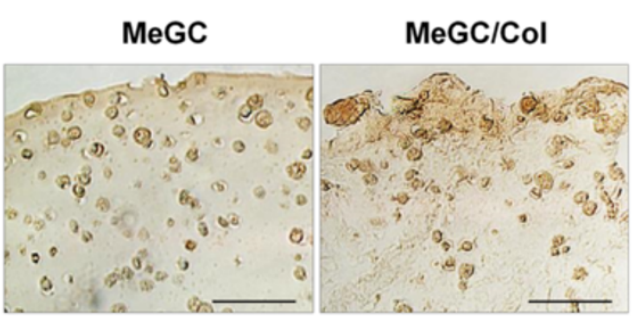

MeGC/Col/TGF

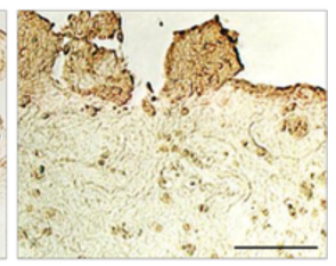

B

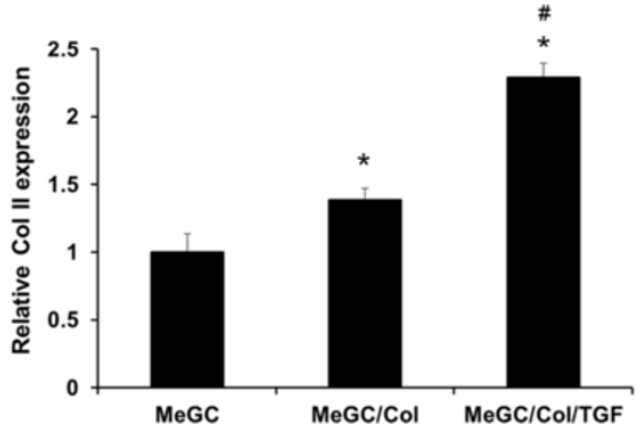

Figure 7 Differentiation of hSMSCs cultured in hydrogels. (A) Type II collagen staining of hSMSCs cultured in hydrogels at 21 days and (B) quantification of Col II staining by image analysis. Scale bar $=100 \mu \mathrm{m}$. (*: $p<0.05$ compared with MeGC and \#: $p<0.05$ compared with MeGC/Col, $\mathrm{n}=3$ ). 


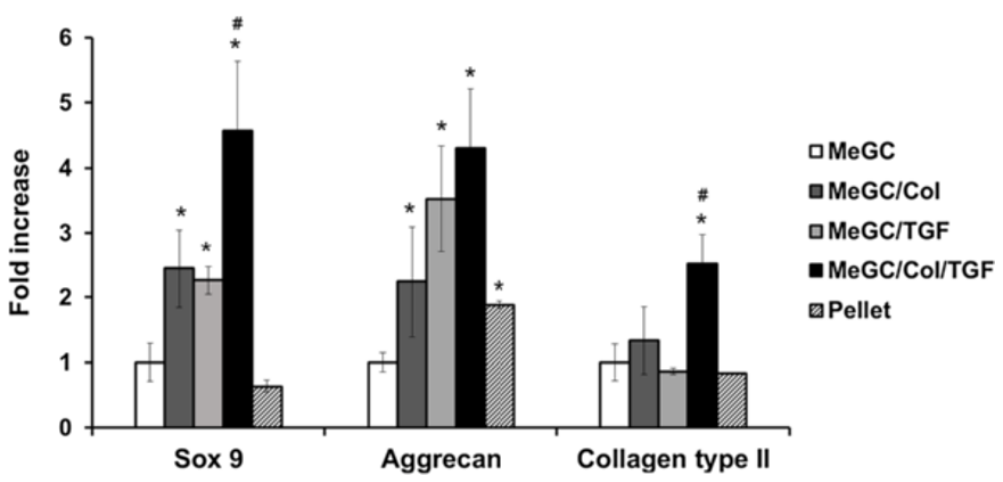

Figure 8 Gene expression of Sox 9, aggrecan, and Collagen type II in hSMSCs cultured in hydrogels or pellets on day 14. $\left(^{*}: p<0.05\right.$ compared with MeGC and \#: $p<0.05$ compared with MeGC/Col or MeGC/TGF, $\mathrm{n}=3$ ).

$[14,40]$. Furthermore, Col II was also impregnated into the hydrogel network to further accelerate chondrogenic differentiation since Col II is a main component in the ECM of articular cartilage [6]. Therefore, in the present study, sophisticated functional scaffolds with a chemical moiety (i.e., SMCC) were designed to eventually match unmet needs for functional cartilage regeneration.

The Col II concentration of $0.4 \%$ was optimized in our previous study to promote chondrogenesis in MeGC hydrogels [41]. Our previous study showed that the incorporation of Col II $(0.4 \% \mathrm{w} / \mathrm{v})$ increased the compressive modulus of the hydrogel from 4.6 to $7.0 \mathrm{kPa}$ [41]. We did not investigate higher concentrations because Col II concentrations over $0.4 \%$ were too viscous to handle and resulted in non-uniform mixing with MeGC. The final concentration of $10 \mu \mathrm{g} / \mathrm{ml}$ was chosen for the current studies because TGF- $\beta 1$ concentrations above $10 \mu \mathrm{g} / \mathrm{ml}$ reduced the mechanical properties of the cross-linked hydrogel. This may be due to the reduced crosslinking density or interactions between polymer chains by the conjugated proteins. Although the mechanical properties of the hydrogels were lower than those of native cartilage, our hydrogel system was designed to mainly serve as an appropriate carrier for stem cells and growth factors, and the degrading hydrogel will be replaced by nascent cartilage tissue in vivo with sufficient mechanical support. Additional studies on the mechanical integrity of the construct in cartilage defects could further enhance the future clinical translation.

Our release data demonstrated the sustained release of TGF- $\beta 1$ chemically conjugated on hydrogel via an SMCC crosslinker. The observed release may be due to hydrolysis of covalent linkages in the environment of serum-supplemented media that present various proteins, electrolytes, hormones, and enzymes. Similar observations of sustained release have been reported using BMP-2 conjugated on biomaterial surfaces via SMCC $[24,33]$. It is also possible that material surfaces can affect chemical stability of the conjugates and the release pattern. It has been demonstrated that different biomaterials showed distinctive release pattern of covalently bound BMP-2 using the same crosslinker [42], indicating that the substrate material as well as the conjugation strategy can affect the release pattern. Further studies to elucidate the conjugation and release mechanisms are needed to determine appropriate linkage for the TGF- $\beta 1$ conjugation. Although positively charged TGF- $\beta 1(\mathrm{p} I=$ 9.5) can interact electrostatically with Col II, the addition of Col II in MeGC hydrogels did not suppress the release of TGF- $\beta 1$ from the hydrogels. This is possibly due to the additional proteins in serum-supplemented media that abolished the electrostatic interaction effects. Similar observations of increased release by serum proteins have been reported in our previous study using Colcoated scaffolds and cationic histone [43]. In contrast, approximately $70 \%$ of the initially loaded TGF- $\beta 1$ by a non-specific adsorption method was released from the $\mathrm{MeGC} / \mathrm{Col}$ hydrogels during the first week (Additional file 1: Figure S1). We observed a fewer number of cell aggregations in these hydrogels with TGF- $\beta 1$ adsorption compared with the TGF- $\beta 1$-conjugated hydrogels, indicating that the chemical conjugation strategy provides more favorable microenvironment to induce chondrogenesis. Similar release kinetics dependent osteogenic activity was observed in BMP-2 loaded polycaprolactone scaffolds [33]. Previous studies also revealed that sustained release of growth factors to the designated sites may further enhance cellular responses leading to desired morphogenesis of progenitor cells $[44,45]$.

While individual use of SMCC, visible blue light (VBL) and riboflavin (vitamin B2) has proven to be biocompatible $[46,47]$, it is crucial to evaluate the bioactivity of the integrated system all together. The Live/Dead staining images and proliferation data revealed that the encapsulated hSMSCs in MeGC-based hydrogels was highly viable throughout the scaffolds $(>90 \%)$ despite the use of bioconjugation agent (i.e., SMCC). Furthermore, the 
proliferation data showed that the proliferation of hSMCSs in MeGC/Col/TGF was lower compared to the cells in $\mathrm{MeGC/Col}$, suggesting that the conjugated TGF$\beta 1$ on the hydrogels may promote the encapsulated hSMSCs to undergo chondrogenic differentiation after 14 days of culture. However, hSMSCs encapsulated in MeGC/TGF hydrogel displayed a similar proliferation rate to the MeGC group and most of the seeded cells were present as individual cells without forming aggregation over 21 days, indicating that TGF- $\beta 1$-mediated chondrogenesis is not sufficient without collagen interaction. Previous studies demonstrated that chondrogenic differentiation was most predominant in the cells stimulated with TGF- $\beta 1$ in the presence of Col II, indicating synergistic effects of Col II binding and TGF- $\beta 1$ signaling on chondrogenesis [28-30].

It is known that cell-matrix interaction plays important role in cartilage tissue engineering and previous reports suggest that cell morphology depends on scaffold type, composition and architecture $[48,49]$. In cartilage development, the round cell morphology has to maintain for chondrogenic differentiation to promote cartilagespecific ECM production. However, the predominant cell morphology observed on most scaffolds except for hydrogels has been known to be elongated fibroblastic morphology, which triggers cell spreading and fibrous matrix deposition [50]. Thus, it is not surprising to use hydrogels for cartilage tissue engineering ranging from natural hydrogels such as hyaluronic acids to synthetic ones such as polyethylene (PEG)-based hydrogels [51,52]. However, the cells encapsulated in hydrogels often lose their chondrogenic phenotype with elongated morphology, depending on hydrogel types and culture conditions $[47,49]$. Therefore, it is crucial to design appropriate microenvironments to promote and maintain chondrogenic differentiation with round (or spherical) cell morphology during cell growth [53].

Cell-cell interactions are important steps in mediating an early mesenchymal condensation and chondrogenesis. The condensation events are known to be initiated by cell-cell adhesion mediated by cell adhesion molecules (N-cadherin, N-CAM) as well as cell-matrix interactions (collagen, aggrecan) [54-57]. Collagen has been shown to induce mesenchymal condensation and subsequent chondrogenesis via integrin-mediated cell adhesion by enhancing cell-matrix interactions [58-60]. Chondrogenic differentiation is further modified by the involvement of various growth factors (TGF- $\beta$, Wnt, fibroblast growth factors) that initiate intracellular signaling pathways transduced by protein kinase $\mathrm{C}$ and mitogenactivated protein kinases $[28,61,62]$. One of the most encouraging findings in the present study is that MeGC hydrogels supported close cell-cell contact and round cell morphology maintained throughout the culture periods with increased ECM production such as GAG and Col II expression over time. Moreover, Col II impregnation did not compromise cell morphology despite its fibrous nature. It is possible to speculate that cell morphology of the encapsulated hSMSCs maintained spherical morphology since their size is greater than that of collagen fibers [48]. Furthermore, we demonstrated that incorporation of Col II and TGF- $\beta 1$ into the MeGC hydrogels at least additively increased density of cell clusters and cartilage-specific ECM production in the hydrogels. Given that the Col II initially incorporated in the hydrogel was obtained from chicken sternal cartilage and the primary antibody used for IHC has very little cross-reactivity with chicken, the observed Col II staining indicates cartilaginous matrix produced by the encapsulated cells, not the Col II initially added in the hydrogel.

There has been a controversy whether exogenous scaffolds are necessary for cartilage development as opposed to bone regeneration, where a general consensus that exogenous three dimensional (3D) scaffolds are required for the tissue development $[12,63,64]$. However, it is our point of view and others' as well that 'functional' 3D scaffolds may be needed for cartilage tissue engineering to immobilize implanted cells in the defect site and to facilitate ECM deposition via cell-scaffold interactions during the tissue development [65]. Overall, MeGC hydrogels supplemented with Col II impregnation and TGF- $\beta 1$ bioconjugation may be an attractive synthetic ECM for cartilage tissue regeneration.

\section{Conclusions}

To enhance chondrogenic differentiation of encapsulated cells, functional chitosan hydrogels were developed by incorporating TGF- $\beta 1$ and nanofibrous Col II into photocrosslinkable MeGC. Controlled delivery of TGF$\beta 1$ with reduced burst release was demonstrated via bioconjugation of the protein to the hydrogels. The hydrogel system did not compromise viability of encapsulated hSMSCs and the addition of Col II and TGF- $\beta 1$ promoted cellular aggregation and chondrogenic differentiation of the encapsulated cells. This MeGC-based hydrogel system provides a specific microenvironment tailored to promote chondrogenesis in the treatment of cartilage defects.

\section{Materials and methods \\ Materials}

Glycol chitosan (GC: molecular weight $\sim 500 \mathrm{kDa}$ ), glycidyl methacrylate, riboflavin (RF) sodium salt, and type II collagen (Col II) purified from chicken sternal cartilage were all purchased from Sigma-Aldrich (MO, USA) and used as received. Succinimidyl-4-(N-maleimidomethyl) cyclohexane-1-carboxylate (SMCC) was purchased from Pierce (Rockford, IL). Recombinant human TGF- $\beta 1$ was obtained from PeproTech (Rocky Hill, NJ). Human 
SMSCs were provided from Dr. Denis Evseenko (UCLA Orthopedic Surgery).

\section{Preparation of photocrosslinkable hydrogels}

Methacrylated glycol chitosan (MeGC) was prepared as previously reported $[10,47]$. Briefly, glycidyl Methacrylate was added to an aqueous solution of $2 \%(\mathrm{w} / \mathrm{v})$ glycol chitosan ( $\mathrm{pH}$ 9.0) with 1.1 molar ratio of glycidyl methacrylate to the primary amine groups in chitosan then proceed to react via gentle shaking at room temperature for $36 \mathrm{~h}$. The reaction mixture was then neutralized, dialyzed against water for $15 \mathrm{~h}$ using membrane with cutoff molecular weight of $50 \mathrm{kDa}$, and lyophilized for further studies. The degree of methacrylation to the GC was $26 \%$ as determined via ${ }^{1} \mathrm{H}-\mathrm{NMR}$.

The composite solution of $2 \% \mathrm{w} / \mathrm{v}$ MeGC containing $0.4 \% \mathrm{w} / \mathrm{v} \mathrm{Col}(\mathrm{MeGC} / \mathrm{Col})$ was prepared by mixing stock MeGC solution ( $4 \% \mathrm{w} / \mathrm{v}$, in PBS) with $\mathrm{Col}(1.0 \% \mathrm{w} / \mathrm{v}$, in $0.05 \%$ acetic acid). Pure MeGC solution ( $2 \% \mathrm{w} / \mathrm{v})$ was prepared by diluting the $4 \% \mathrm{w} / \mathrm{v}$ MeGC in PBS. The hydrogel was formed by exposing the solution to visible blue light (400-500 nm, 500-600 mW/ $\mathrm{cm}^{2}$, Bisco Inc., Schaumburg, IL) in the presence of RF photoinitiators (6 $\mu \mathrm{M})$.

The interior morphology of hydrogels was observed using scanning electron microscopy (SEM, Nova NanoSEM 230, FEI, Hillsboro, OR). The hydrogels were fixed with $2.5 \%$ glutaraldehyde for $2 \mathrm{~h}$ at room temperature and interior morphology was imaged in low vacuum mode.

\section{TGF- $\beta 1$ conjugation}

TGF- $\beta 1$ was covalently conjugated to $\mathrm{MeGC}$ via SMCC linker. Briefly, $20 \mu \mathrm{L}$ of SMCC $(7.4 \mathrm{mg} / \mathrm{mL})$ was added to $10 \mathrm{~mL}$ of MeGC (2\% w/v in PBS) and incubated for $15 \mathrm{~h}$ at room temperature with gentle shaking. The reaction mixture was then dialyzed against water and lyophilized to receive MeGC-SMCC. To conjugate TGF- $\beta 1$ to MeGC-SMCC, $10 \mu \mathrm{g}$ of TGF- $\beta 1$ in PBS was reacted with $2 \mathrm{~mL}$ of MeGC-SMCC aqueous solution (1\% w/v). Reaction was performed for $15 \mathrm{~h}$ under mild shaking at $4^{\circ} \mathrm{C}$ and then purified with ultrafiltration tubes (MWCO $100 \mathrm{kDa}$ ) according to the manufacturer's manual (Millipore). The concentrated product was recovered, lyophilized, and stored at $-20^{\circ} \mathrm{C}$ for further study.

To examine the release kinetics of proteins, TGF- $\beta 1$ tethered hydrogels (MeGC/Col/TGF) containing cells were prepared as described above and the obtained hydrogels were incubated in DMEM supplemented with $10 \%$ fetal bovine serum (FBS) at $37^{\circ} \mathrm{C}$. The final concentration of TGF- $\beta 1$ in hydrogels was $10 \mu \mathrm{g} / \mathrm{mL}$. The incubating medium was replaced with fresh medium at the designated time interval. The amount of released TGF$\beta 1$ in the medium was measured by using an enzymelinked immunosorbent assay (ELISA) kit (R\&D systems,
Minneapolis, MN). Measurements were performed in triplicate, and the amount of protein release was expressed as a percentage of the initial amount of incorporated protein.

\section{Culture of cells in hydrogels}

Human SMSCs were expanded in DMEM with 20\% FBS, $100 \mu \mathrm{g} / \mathrm{mL}$ streptomycin, and $100 \mathrm{U} / \mathrm{mL}$ penicillin at $37^{\circ} \mathrm{C}$ in a $5 \% \mathrm{CO}_{2}$ humidified atmosphere. SMSCs (passage 3-4) were suspended in $40 \mu \mathrm{l}$ of MeGC, $\mathrm{MeGC} / \mathrm{Col}$, and $\mathrm{MeGC} / \mathrm{Col} / \mathrm{TGF}-\beta 1$ solutions at a density of $10 \times 10^{6}$ cells $/ \mathrm{mL}$ (final concentration of TGF- $\beta 1$ was $10 \mu \mathrm{g} / \mathrm{mL})$. The hydrogels were cultured in chondrogenic medium consisting of DMEM with 10\% FBS, ITS+ Premix supplement (BD Biosciences, Bedford, MA), $100 \mathrm{nM}$ dexamethasone, $40 \mu \mathrm{g} / \mathrm{mL}$ L-proline, $1 \mathrm{mM}$ sodium pyruvate, and $50 \mu \mathrm{g} / \mathrm{mL} \mathrm{L}$-ascorbic acid 2-phosphate (all Sigma-Aldrich) for up to 21 days and the medium was replaced twice a week.

The growth of SMSCs in the hydrogels was observed using a light microscope (Olympus IX71, Olympus, Lake Success, NY). Images of SMSCs in the hydrogels were captured from three randomly chosen fields. The cluster area was quantified from captured images using NIH-Image J software (http:/rsb.info.nih.gov/ij/). Ten to twenty clusters were counted for each hydrogel sample. Proliferation of cells was measured using Cell Counting Kit-8 (CCK-8, Dojindo, Kumamoto, Japan) according to the manufacturer's protocol. To observe the cell viability, cell/hydrogels constructs were washed once with PBS and stained with calcein/ethidium homodimer using a LIVE/DEAD assay kit (Invitrogen, Carlsbad, CA) at $37^{\circ} \mathrm{C}$ for $30 \mathrm{~min}$. Stained samples were observed by a fluorescent microscopy (Olympus IX71 microscope). The percentage viability was determined by calculating the number of live cells (green) normalized to the total number of cells (green and red). All the experiments were performed in triplicate ( $n=3$ per group).

\section{Histological and immunohistochemical analyses}

For histological analysis, cultured hydrogels were fixed with $10 \%$ neutral buffered formalin, embedded in paraffin, then sectioned at $5 \mu \mathrm{m}$. The sections were deparaffinized then stained with hematoxylin and eosin $(\mathrm{H} \& \mathrm{E})$ to examine cellular distribution and morphology. Safranin-O staining was performed to assess glycosaminoglycans (GAG) synthesis. Immunohistochemistry (IHC) was performed to determine Col synthesis. Briefly, sections were incubated with primary antibody against Col (anti-human Col; EMD Millipore, Billerica, MA) and antibody was detected using the SuperPicture ${ }^{\mathrm{TM}}$ polymer detection kit with DAB substrate (Invitrogen) per the manufacturers' instructions. Images were obtained using Olympus IX71 microscope. GAG and Col production was quantified by image analysis of three 
randomly selected fields of each of three Safranin-O staining or Col IHC samples $(n=9 /$ group) relatively quantified by using NIH-ImageJ software.

\section{RNA extraction and quantitative real-time polymerase chain reaction ( $q R T-P C R$ )}

To investigate the gene expressions of SMSCs in the hydrogels, SMSCs-laden MeGC, MeGC/Col, MeGC/ TGF, and MeGC/Col/TGF- $\beta 1$ hydrogels were cultured in chondrogenic media for 14 days. Total RNA was extracted using Trizol reagent and RNeasy Mini Plant kit (Qiagen, Valencia, CA) as previously described [66]. Briefly, $0.5 \mu \mathrm{g}$ of total RNA was reversely transcribed to cDNA using a cDNA transcription kit. The expressions of chondrogenic gene markers Sox 9, aggrecan, and Col II were measured by quantitative real-time PCR using LightCycler 480 PCR (Indianapolis, IN) with $20 \mu \mathrm{l} \mathrm{SYBR}$ Green reaction volume. SMSCs cultured in pellets were used as a standard of comparison. SMSC pellets were prepared as previously described [36] and cultured in chondrogenic medium supplemented with $10 \mathrm{ng} / \mathrm{mL}$ TGF- $\beta 1$. The primers were designed as previously described [67]. The levels of gene expression were normalized with GAPDH. The amount of mRNA expression was expressed as a ratio to the MeGC hydrogel.

\section{Statistical analysis}

Statistical analysis was performed using one way analysis of variances (ANOVA) followed by Tukey's post hoc test. A value of $p<0.05$ was considered statistically significant.

\section{Additional files}

Additional file 1: Figure S1. Supplementary data of TGF- $\beta 1$ release (A) and SMSC growth (B) in MeGC/Col containing non-specifically adsorbed TGF- $\beta 1$. Scale bar $=200 \mu \mathrm{m}$.

Additional file 2: Figure S2. Supplementary data of SMSC cultured in MeGC/TGF without collagen at day 21 including (A) Bright field images, (B) live/dead staining, (C) H \& E staining, and (D) Safranin-O staining. Scale bar $=100 \mu \mathrm{m}$.

\section{Competing interests}

The authors declare that they have no competing interests.

\section{Authors' contributions}

JK participated in data interpretation and discussions, and drafted the manuscript. BL prepared the samples, carried out the experiments, and drafted the manuscript. SK carried out gene expression and release experiments. BC carried out sample characterization and release experiments. DE performed cell isolation and drafted the manuscript. ML conceived and designed the experiments, and drafted the manuscript. All authors read and approved the final manuscript.

\section{Acknowledgements}

This work was supported by the UCLA Academic Senate Research Award and UCLA School of Dentistry Faculty seed grant.

\section{Author details}

'Department of Bio and Chemical Engineering, Hongik University, Sejong 339-701, South Korea. '2Division of Advanced Prosthodontics, University of California, Los Angeles, CA 90095, USA. ${ }^{3}$ Department of Bioengineering, University of California, Los Angeles, CA 90095, USA. ${ }^{4}$ Department of Orthopaedic Surgery, University of California, Los Angeles, CA 90095, USA.

Received: 3 October 2014 Accepted: 24 December 2014

Published: 14 January 2015

\section{References}

1. Mehrotra C, Remington PL, Naimi TS, Washington W, Miller R. Trends in total knee replacement surgeries and implications for public health, 1990-2000. Public Health Rep. 2005;120:278-82.

2. Sasso RC, LeHuec JC, Shaffrey C, Grp SIR. lliac crest bone graft donor site pain after anterior lumbar interbody fusion - A prospective patient satisfaction outcome assessment. J Spinal Disord Tech. 2005;18:S77-81.

3. Sasso RC, LeHuec JC, Shaffrey Cl. Iliac crest graft site pain after anterior lumbar interbody fusion: a prospective patient outcome assessment. Neurosurgery. 2002:51:581-1.

4. Benya PD, Padilla SR, Nimni ME. Independent regulation of collagen types by chondrocytes during the loss of differentiated function in culture. Cell. 1978;15:1313-21.

5. Diaz-Romero J, Gaillard JP, Grogan SP, Nesic D, Trub T, Mainil-Varlet P. Immunophenotypic analysis of human articular chondrocytes: changes in surface markers associated with cell expansion in monolayer culture. J Cell Physiol. 2005;202:731-42.

6. Ahmed TA, Hincke MT. Strategies for articular cartilage lesion repair and functional restoration. Tissue Eng Part B Rev. 2010;16:305-29.

7. Noth U, Rackwitz L, Heymer A, Weber M, Baumann B, Steinert A, et al. Chondrogenic differentiation of human mesenchymal stem cells in collagen type I hydrogels. J Biomed Mater Res A. 2007;83:626-35.

8. Kim IL, Mauck RL, Burdick JA. Hydrogel design for cartilage tissue engineering: a case study with hyaluronic acid. Biomaterials. 2011;32:8771-82.

9. Noth U, Rackwitz L, Steinert AF, Tuan RS. Cell delivery therapeutics for musculoskeletal regeneration. Adv Drug Deliver Rev. 2010;62:765-83.

10. Hu J, Hou Y, Park H, Choi B, Hou S, Chung A, et al. Visible light crosslinkable chitosan hydrogels for tissue engineering. Acta Biomater. 2012;8:1730-8.

11. Park H, Choi B, Hu J, Lee M. Injectable chitosan hyaluronic acid hydrogels for cartilage tissue engineering. Acta Biomater. 2013;9:4779-86.

12. Huey DJ, Hu JC, Athanasiou KA. Unlike bone, cartilage regeneration remains elusive. Science. 2012;338:917-21.

13. Natoli RM, Revell CM, Athanasiou KA. Chondroitinase ABC treatment results in greater tensile properties of self-assembled tissue-engineered articular cartilage. Tissue Eng Part A. 2009;15:3119-28.

14. $\mathrm{Ha}$ CW, Noh MJ, Choi KB, Lee KH. Initial phase I safety of retrovirally transduced human chondrocytes expressing transforming growth factor-beta-1 in degenerative arthritis patients. Cytotherapy. 2012;14:247-56.

15. van Lent PL, Blom AB, van der Kraan P, Holthuysen AE, Vitters E, van Rooijen N, et al. Crucial role of synovial lining macrophages in the promotion of transforming growth factor beta-mediated osteophyte formation. Arthritis Rheum. 2004:50:103-11.

16. van Beuningen HM, Glansbeek HL, van der Kraan PM, van den Berg WB. Differential effects of local application of BMP-2 or TGF-beta 1 on both articular cartilage composition and osteophyte formation. Osteoarthritis Cartilage. 1998;6:306-17.

17. McCaffrey TA, Falcone DJ, Vicente D, Du B, Consigli S, Borth W. Protection of transforming growth factor-beta 1 activity by heparin and fucoidan. J Cell Physiol. 1994;159:51-9.

18. Lyon M, Rushton G, Gallagher JT. The interaction of the transforming growth factor-betas with heparin/heparan sulfate is isoform-specific. J Biol Chem. 1997;272:18000-6.

19. McCaffrey TA, Falcone DJ, Borth W, Weksler BB. Alpha 2-macroglobulin/ transforming growth factor-beta 1 interactions. Modulation by heparin-like molecules and effects on vascular smooth muscle cells. Ann N Y Acad Sci. 1994;737:368-82

20. McCaffrey TA, Falcone DJ, Du B. Transforming growth factor-beta 1 is a heparin-binding protein: identification of putative heparin-binding regions and isolation of heparins with varying affinity for TGF-beta 1. J Cell Physiol. 1992;152:430-40. 
21. Park JS, Woo DG, Yang HN, Lim HJ, Chung HM, Park KH. Heparin-bound transforming growth factor-beta3 enhances neocartilage formation by rabbit mesenchymal stem cells. Transplantation. 2008;85:589-96.

22. Park JS, Woo DG, Yang HN, Na K, Park KH. Transforming growth factor beta-3 bound with sulfate polysaccharide in synthetic extracellular matrix enhanced the biological activities for neocartilage formation in vivo. J Biomed Mater Res A. 2009;91:408-15.

23. Jung HH, Park K, Han DK. Preparation of TGF-beta1-conjugated biodegradable pluronic F127 hydrogel and its application with adipose-derived stem cells. J Control Release. 2010;147:84-91.

24. Zhang Q, He QF, Zhang TH, Yu XL, Liu Q, Deng FL. Improvement in the delivery system of bone morphogenetic protein-2: a new approach to promote bone formation. Biomed Mater. 2012;7:045002.

25. Bessa PC, Casal M, Reis RL. Bone morphogenetic proteins in tissue engineering: the road from laboratory to clinic, part II (BMP delivery). J Tissue Eng Regen Med. 2008;2:81-96.

26. Zhang L, Yuan T, Guo L, Zhang X. An in vitro study of collagen hydrogel to induce the chondrogenic differentiation of mesenchymal stem cells. J Biomed Mater Res A. 2012;100:2717-25.

27. Pabbruwe MB, Kafienah W, Tarlton JF, Mistry S, Fox DJ, Hollander AP. Repair of meniscal cartilage white zone tears using a stem cell/collagen-scaffold implant. Biomaterials. 2010;31:2583-91.

28. Schneiderbauer MM, Dutton CM, Scully SP. Signaling "cross-talk" between TGF-beta1 and ECM signals in chondrocytic cells. Cell Signal. 2004;16:1133-40.

29. Bosnakovski D, Mizuno M, Kim G, Takagi S, Okumura M, Fujinaga T. Chondrogenic differentiation of bovine bone marrow mesenchymal stem cells (MSCS) in different hydrogels: influence of collagen type II extracellular matrix on MSC chondrogenesis. Biotechnol Bioeng. 2006;93:1152-63.

30. Warstat K, Meckbach D, Weis-Klemm M, Hack A, Klein G, de Zwart P, et al. TGF-beta enhances the integrin alpha2beta1-mediated attachment of mesenchymal stem cells to type I collagen. Stem Cells Dev. 2010;19:645-56.

31. Bieniarz C, Husain M, Barnes G, King CA, Welch CJ. Extended length heterobifunctional coupling agents for protein conjugations. Bioconjug Chem. 1996;7:88-95.

32. Lim EH, Sardinha JP, Myers S, Stevens M. Latent transforming growth factor-beta1 functionalised electrospun scaffolds promote human cartilage differentiation: towards an engineered cartilage construct. Archives of plastic surgery. 2013:40:676-86

33. Zhang H, Migneco F, Lin CY, Hollister SJ. Chemically-conjugated bone morphogenetic protein-2 on three-dimensional polycaprolactone scaffolds stimulates osteogenic activity in bone marrow stromal cells. Tissue Eng Part A. 2010;16:3441-8.

34. He QF, Zhao YN, Chen B, Xiao ZF, Zhang J, Chen L, et al. Improved cellularization and angiogenesis using collagen scaffolds chemically conjugated with vascular endothelial growth factor. Acta Biomater. 2011;7:1084-93.

35. Huang CYC, Reuben PM, D'Ippolito G, Schiller PC, Cheung HS. Chondrogenesis of human bone marrow-derived mesenchymal stem cells in agarose culture. Anat Rec Part A. 2004;278A:428-36.

36. Bosnakovski D, Mizuno M, Kim G, Ishiguro T, Okumura M, Iwanaga T, et al. Chondrogenic differentiation of bovine bone marrow mesenchymal stem cells in pellet cultural system. Exp Hematol. 2004;32:502-9.

37. Zhang LM, Su PQ, Xu CX, Yang JL, Yu WH, Huang DS. Chondrogenic differentiation of human mesenchymal stem cells: a comparison between micromass and pellet culture systems. Biotechnol Lett. 2010;32:1339-46.

38. Cheng NC, Estes BT, Awad HA, Guilak F. Chondrogenic differentiation of adipose-derived adult stem cells by a porous scaffold derived from native articular cartilage extracellular matrix. Tissue Eng Part A. 2009;15:231-41.

39. Diekman BO, Rowland CR, Lennon DP, Caplan Al, Guilak F. Chondrogenesis of adult stem cells from adipose tissue and bone marrow: induction by growth factors and cartilage-derived matrix. Tissue Eng Pt A. 2010;16:523-33.

40. Chung C, Burdick JA. Engineering cartilage tissue. Adv Drug Deliv Rev. 2008;60:243-62.

41. Choi B, Kim S, Lin B, Wu BM, Lee M. Cartilaginous extracellular matrix-modified chitosan hydrogels for cartilage tissue engineering. ACS Appl Mater Interfaces. 2014;6:20110-21.

42. Gharibjanian NA, Chua WC, Dhar S, Scholz T, Shibuya TY, Evans GR, et al. Release kinetics of polymer-bound bone morphogenetic protein-2 and its effects on the osteogenic expression of MC3T3-E1 osteoprecursor cells. Plast Reconstr Surg. 2009;123:1169-77.

43. Lee $M$, Chen TT, Iruela-Arispe ML, Wu BM, Dunn JC. Modulation of protein delivery from modular polymer scaffolds. Biomaterials. 2007;28:1862-70.
44. Lutolf MP, Weber FE, Schmoekel HG, Schense JC, Kohler T, Muller R, et al. Repair of bone defects using synthetic mimetics of collagenous extracellular matrices. Nat Biotechnol. 2003;21:513-8.

45. Boerckel JD, Kolambkar YM, Dupont KM, Uhrig BA, Phelps EA, Stevens HY, et al. Effects of protein dose and delivery system on BMP-mediated bone regeneration. Biomaterials. 2011;32:5241-51.

46. Ibusuki S, Halbesma GJ, Randolph MA, Redmond RW, Kochevar IE, Gill TJ. Photochemically cross-linked collagen gels as three-dimensional scaffolds for tissue engineering. Tissue Eng. 2007;13:1995-2001.

47. Amsden BG, Sukarto A, Knight DK, Shapka SN. Methacrylated glycol chitosan as a photopolymerizable biomaterial. Biomacromolecules. 2007;8:3758-66.

48. Nuernberger S, Cyran N, Albrecht C, Redl H, Vecsei V, Marlovits S. The influence of scaffold architecture on chondrocyte distribution and behavior in matrixassociated chondrocyte transplantation grafts. Biomaterials. 2011;32:1032-40.

49. Awad HA, Wickham MQ, Leddy HA, Gimble JM, Guilak F. Chondrogenic differentiation of adipose-derived adult stem cells in agarose, alginate, and gelatin scaffolds. Biomaterials. 2004;25:3211-22.

50. Dehne T, Karlsson C, Ringe J, Sittinger M, Lindahl A. Chondrogenic differentiation potential of osteoarthritic chondrocytes and their possible use in matrix-associated autologous chondrocyte transplantation. Arthritis Res Ther. 2009;11:R133.

51. Toh WS, Lee EH, Guo XM, Chan JKY, Yeow CH, Choo AB, et al. Cartilage repair using hyaluronan hydrogel-encapsulated human embryonic stem cell-derived chondrogenic cells. Biomaterials. 2010;31:6968-80.

52. Hoffman AS. Hydrogels for biomedical applications. Adv Drug Deliver Rev. 2002;54:3-12.

53. Hutmacher DW. Scaffolds in tissue engineering bone and cartilage. Biomaterials. 2000;21:2529-43.

54. Cushing MC, Anseth KS. Materials science. Hydrogel cell cultures. Science. 2007;316:1133-4.

55. DeLise AM, Fischer L, Tuan RS. Cellular interactions and signaling in cartilage development. Osteoarthritis and cartilage/OARS, Osteoarthritis Research Society. 2000:8:309-34.

56. Tacchetti C, Tavella S, Dozin B, Quarto R, Robino G, Cancedda R. Cell condensation in chondrogenic differentiation. Exp Cell Res. 1992;200:26-33.

57. Hall BK, Miyake T. All for one and one for all: condensations and the initiation of skeletal development. Bioessays. 2000;22:138-47.

58. Lu ZF, Doulabi BZ, Wuisman PI, Bank RA, Helder MN. Influence of collagen type II and nucleus pulposus cells on aggregation and differentiation of adipose tissue-derived stem cells. J Cell Mol Med. 2008;12:2812-22.

59. Jurgens WJ, Lu Z, Zandieh-Doulabi B, Kuik DJ, Ritt MJ, Helder MN. Hyperosmolarity and hypoxia induce chondrogenesis of adipose-derived stem cells in a collagen type 2 hydrogel. J Tissue Eng Regen Med. 2012;6:570-8.

60. Yu SM, Li Y, Kim D. Collagen mimetic peptides: progress towards functional applications. Soft Matter. 2011;7:7927-38.

61. Chun JS, Oh H, Yang S, Park M. Wnt signaling in cartilage development and degeneration. BMB Rep. 2008;41:485-94.

62. Yang G, Yang X. Roles of TGF-b superfamily in the genesis, development and maintenance of cartilage. Yi chuan = Hereditas/Zhongguo yi chuan xue hui bian ji. 2008;30:953-9.

63. Schubert T, Anders S, Neumann E, Scholmerich J, Hofstadter F, Grifka J, et al. Long-term effects of chondrospheres on cartilage lesions in an autologous chondrocyte implantation model as investigated in the SCID mouse model. Int J Mol Med. 2009;23:455-60.

64. Lewis PB, McCarty 3rd LP, Yao JQ, Williams JM, Kang R, Cole BJ. Fixation of tissue-engineered human neocartilage constructs with human fibrin in a caprine model. J Knee Surg. 2009:22:196-204.

65. Chung C, Burdick JA. Influence of three-dimensional hyaluronic acid microenvironments on mesenchymal stem cell chondrogenesis. Tissue Eng Part A. 2009;15:243-54

66. Fan JB, Park H, Tan S, Lee M. Enhanced osteogenesis of adipose derived stem cells with noggin suppression and delivery of BMP-2. Plos One. 2013;8:e72474

67. Liu SQ, Tian QA, Hedrick JL, Hui JHP, Ee PLR, Yang YY. Biomimetic hydrogels for chondrogenic differentiation of human mesenchymal stem cells to neocartilage. Biomaterials. 2010;31:7298-307.

doi:10.1186/1754-1611-9-1

Cite this article as: Kim et al:: TGF- $\beta 1$ conjugated chitosan collagen hydrogels induce chondrogenic differentiation of human synovium-derived stem cells. Journal of Biological Engineering 2015 9:1. 